\title{
Energy supply for vehicles - can it be regenerative?
}

Lars Mönch,

Co-Author: Dr. Martin Lange,

both German Federal Environment Agency

Zum Zeitpunkt der Drucklegung lagen diese Unterlagen noch nicht vor. Wir bitten um Verständnis. 